\title{
Conditional evolution in single-atom cavity QED
}

\author{
Andrei N. Soklakov* and Rüdiger Schack
}

(25 April 2001)

\begin{abstract}
We consider a typical setup of cavity QED consisting of a two-level atom interacting strongly with a single resonant electromagnetic field mode inside a cavity. The cavity is resonantly driven and the output undergoes continuous homodyne measurements. We derive an explicit expression for the state of the system conditional on a discrete photocount record. This expression takes a particularly simple form if the system is initially in the steady state. As a byproduct, we derive a general formula for the steady state that had been conjectured before in the strong driving limit.
\end{abstract}

\section{INTRODUCTION}

Recently there has been much experimental progress in single-atom cavity QED [1,2]. In addition to their inherent fundamental importance, these experiments provide insight into the physics of open quantum systems, with potential applications to, e.g., quantum chaos [3], quantum control [4], and quantum computing [5].

In this article we consider a typical experimental setup of single-atom cavity QED [6], as illustrated in Fig. 1. The setup consists of a single two-level atom located inside a high finesse optical cavity, which is externally driven. A set of photodetectors is arranged to monitor the field escaping from the system into the environment. We assume that the leakage of photons from the cavity mode through an output mirror is the only significant channel through which the system interacts with the environment. This assumption can be very realistic for high-finesse cavities [7]. Also, for simplicity, we adjust the cavity length and the frequency of the driving field so that they both coincide with the frequency of the atomic transition. The cavity output is monitored using continuous homodyne measurements [8]. These measurements are parameterized by one complex parameter: the reference field $\beta$ which is added to the cavity output on a beam-splitter prior to the detection.

Given the output of the photodetectors, it is possible in principle to write the conditional quantum state inside the cavity as a function of time and the measurement record. Usually, the conditional state is computed numerically [9, 10] using the formalism of stochastic master equations [11,12]; these numerical computations can require very large computational resources. For some experiments, however, the ability to process data in real time is crucial [四]. It is therefore important to develop analytical tools for conditional state evolution.

In this paper we derive explicit expressions for the state conditioned on a discrete homodyne measurement record in the strong coupling regime, where the atom is strongly coupled to the intracavity field. Our calculations are valid for arbitrary driving field strengths. Our results are applicable to experiments such as the atomic cavity microscope [1,2], where the strong coupling is essential, but strong driving leads to the problem of saturation [7]. We

*Direct any correspondence to: a.soklakov@rhul.ac.uk 
give special attention to the experimentally important case that the system is initially in the steady state.

The paper is organized as follows. In Sec. II we describe the equations that model the physical system. In Sec. III we review the formalism of conditional quantum evolution and introduce the approximations for the strong coupling regime. In Sec. IV we derive a general formula for the system state conditioned on a discrete photocount record, for an arbitrary initial system state. In Sec. $\nabla$, we give a derivation of a general expression for the steady state. In Sec. VI we find simple formulas for the conditional evolution in the case that the system is initially in the steady state. We conclude in Sec. VII.

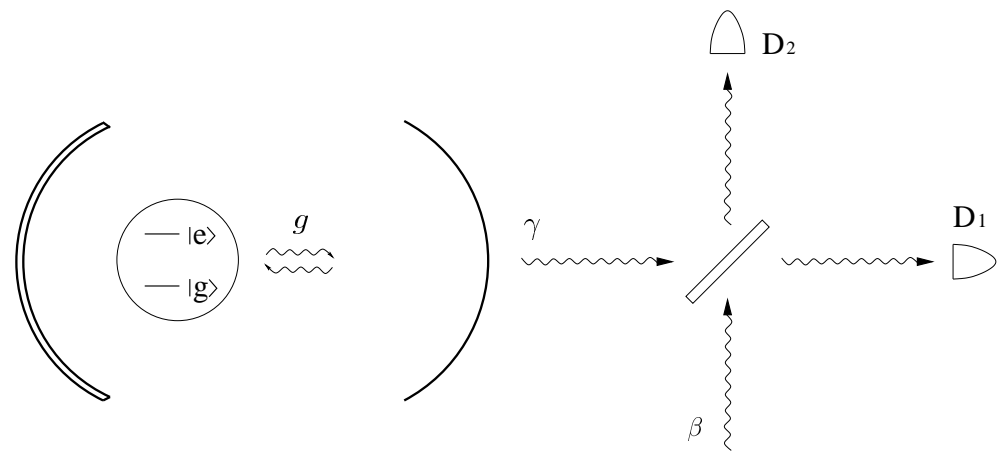

FIG. 1. Homodyne measurements in cavity QED. Basic parameters of the system are the strength of the atom-cavity coupling $g$, and the cavity field decay rate $\gamma$. The cavity is resonantly driven by an external laser field $E$, and the cavity output field is analyzed by the detectors $D_{1}$ and $D_{2}$ after being added to the reference field $\beta$ on the beam-splitter.

\section{MATHEMATICAL MODEL AND MAIN APPROXIMATIONS}

Let $|g\rangle$ and $|e\rangle$ be the ground and excited states of the atom. For simplicity, we choose the cavity length so that the frequency of the resonant optical mode coincides with the frequency of the atomic transition. Using the dipole and the rotating wave approximations the interaction of the two-level atom with the electromagnetic field inside the cavity is described by the Hamiltonian 13

$$
H_{\mathrm{int}} \equiv i g\left(a^{\dagger} \sigma-a \sigma^{\dagger}\right)
$$

where $\sigma=|\mathrm{g}\rangle\langle\mathrm{e}|, g$ is the strength of the atom-cavity coupling, and $a$ is the annihilation operator for the intracavity field. Including dissipation and on-resonant driving of the cavity mode, the total unconditional master equation in a frame rotating at the driving laser frequency reads

$$
\dot{\rho}=\left[-i H_{\mathrm{int}}+E\left(a^{\dagger}-a\right), \rho\right]+\frac{\gamma}{2}\left(2 a \rho a^{\dagger}-a^{\dagger} a \rho-\rho a^{\dagger} a\right),
$$

where $\rho$ is the joint density operator for the atom and the intracavity field, $E$ is the strength of the driving, and $\gamma$ is the rate of energy loss due to the leakage of photons from the cavity mode through an output mirror.

From the experimental point of view the question of the steady state is very important. In fact, using contemporary techniques it is very difficult to prepare the system in question 
in any other state. Using the Jaynes-Cummings model, Alsing and Carmichael [9] have shown numerically that in the strong driving limit $E \gg g$ the system approaches a steady state of the form

$$
\rho_{\mathrm{ss}}=\frac{1}{2}\left(|\alpha ;+\rangle\left\langle\alpha ;+|+| \alpha^{*} ;-\right\rangle\left\langle\alpha^{*} ;-\right|\right),
$$

where $|\alpha ;+\rangle$ and $\left|\alpha^{*} ;-\right\rangle$ are two orthogonal quantum states

$$
\begin{gathered}
|\alpha ;+\rangle=\frac{1}{\sqrt{2}}|\alpha\rangle(|\mathrm{g}\rangle+i|\mathrm{e}\rangle) \equiv|\alpha\rangle|+\rangle, \\
\left|\alpha^{*} ;-\right\rangle=\frac{1}{\sqrt{2}}\left|\alpha^{*}\right\rangle(|\mathrm{g}\rangle-i|\mathrm{e}\rangle) \equiv|\alpha\rangle|-\rangle,
\end{gathered}
$$

and where $|\alpha\rangle$ is the coherent field state with amplitude

$$
\alpha=(2 E+i g) / \gamma
$$

This result has been confirmed in a more recent numerical simulation [10. Using matrix notation for the intra-atomic degrees of freedom in the basis $\{| \pm\rangle\}$, Eq. (3) can be rewritten in the convenient form

$$
\rho_{\mathrm{ss}}=\frac{1}{2}\left(\begin{array}{cc}
|\alpha\rangle\langle\alpha| & 0 \\
0 & \left|\alpha^{*}\right\rangle\left\langle\alpha^{*}\right|
\end{array}\right)
$$

which will be useful below.

In this paper, we work in the strong-coupling regime $(g \gg \gamma)$, which justifies considering the evolution on time scales large compared to $1 / \mathrm{g}$. In Sec. $\mathrm{V}$ we give an analytical proof that on those timescales, Eq. (3) is a steady state of Eq. (2), for arbitrary values of the driving $E$.

\section{THE MEASUREMENT}

We now rewrite Eq. (2) in the form

$$
\dot{\rho}=\mathcal{L} \rho,
$$

where the superoperator $\mathcal{L}$ is defined as

$$
\mathcal{L} \rho \equiv\left[-i H_{\mathrm{int}}+E\left(a^{\dagger}-a\right), \rho\right]+\frac{\gamma}{2}\left(2 a \rho a^{\dagger}-a^{\dagger} a \rho-\rho a^{\dagger} a\right) .
$$

Let the initial condition be $\rho(0)=\rho_{0}$. Given superoperators $\mathcal{S}_{0}, \mathcal{J}_{1}$ and $\mathcal{J}_{2}$ such that

$$
\mathcal{S}_{0}(t)=e^{\left(\mathcal{L}-\mathcal{J}_{1}-\mathcal{J}_{2}\right) t},
$$

the solution to Eq. (7) can be written using a Dyson expansion,

$$
\rho(\Delta t)=\sum_{m=0}^{\infty} \sum_{k_{1}, \ldots, k_{m}} p\left(k_{1}, \ldots, k_{m} ; \Delta t\right) \rho_{\mathrm{c}}\left(k_{1}, \ldots, k_{m} ; \Delta t\right)
$$


where $\operatorname{tr} \rho_{\mathrm{c}}\left(k_{1}, \ldots, k_{m} ; \Delta t\right)=1$ and

$$
\begin{aligned}
& p\left(k_{1}, \ldots, k_{m} ; \Delta t\right) \rho_{\mathrm{c}}\left(k_{1}, \ldots, k_{m} ; \Delta t\right)= \\
& \int_{0}^{\Delta t} d t_{m} \cdots \int_{0}^{t_{3}} d t_{2} \int_{0}^{t_{2}} d t_{1} \mathcal{S}_{0}\left(\Delta t-t_{m}\right) \mathcal{J}_{k_{m}} \mathcal{S}_{0}\left(t_{m}-t_{m-1}\right) \mathcal{J}_{k_{m-1}} \cdots \mathcal{S}_{0}\left(t_{1}\right) \rho_{0} .
\end{aligned}
$$

Following [11,12 we define the "smooth evolution" operator $\mathcal{S}_{0}$ as

$$
\mathcal{S}_{0}(t) \rho \equiv N_{0}(t) \rho\left[N_{0}(t)\right]^{\dagger},
$$

where

$$
N_{0}(t) \equiv \exp \left[-i H_{\mathrm{int}} t+E\left(a^{\dagger}-a\right) t-\frac{\gamma}{2}\left(a^{\dagger} a+|\beta|^{2}\right) t\right],
$$

and the "jump" operators $\mathcal{J}_{1}$ and $\mathcal{J}_{2}$ as

$$
\mathcal{J}_{k} \rho \equiv C_{k} \rho C_{k}^{\dagger}, \quad \text { where } \quad C_{k} \equiv \sqrt{\gamma / 2} e^{i \pi(k-1) / 2}\left[a+(-1)^{k} \beta\right]
$$

The following lemma, included for completeness, shows that the definitions of $\mathcal{S}_{0}, \mathcal{J}_{1}$ and $\mathcal{J}_{2}$ just given are consistent with Eq. (9).

Lemma 1 The above definitions satisfy the requirement

$$
\mathcal{S}_{0}(t)=e^{\left(\mathcal{L}-\mathcal{J}_{1}-\mathcal{J}_{2}\right) t}
$$

and therefore Eqns. (10) and (11) indeed give a solution to (17).

Proof

Keeping terms to first order in $\tau$ we have

$$
\begin{aligned}
\mathcal{S}_{0}(\tau) \rho= & N_{0}(\tau) \rho\left[N_{0}(\tau)\right]^{\dagger} \\
= & \rho+\left(\left[-i H_{\mathrm{int}}+E\left(a^{\dagger}-a\right), \rho\right]\right. \\
& \left.\quad-\frac{\gamma}{2}\left(a^{\dagger} a \rho+\rho a^{\dagger} a\right)-\gamma|\beta|^{2} \rho\right) \tau+O\left(\tau^{2}\right) \\
& =(\mathbb{1}+\tau \cdot \mathcal{L}) \rho-\gamma\left(a \rho a^{\dagger}+|\beta|^{2} \rho\right) \tau+O\left(\tau^{2}\right) .
\end{aligned}
$$

On the other hand by direct calculation we have

$$
\mathcal{J}_{k} \rho=\frac{\gamma}{2}\left[a \rho a^{\dagger}+(-1)^{k}\left(\beta \rho a^{\dagger}+\beta^{*} a \rho\right)+|\beta|^{2} \rho\right]
$$

which implies that

$$
\left(\mathcal{J}_{1}+\mathcal{J}_{2}\right) \rho=\gamma\left(a \rho a^{\dagger}+|\beta|^{2} \rho\right)
$$

Equation (16) therefore becomes

$$
\mathcal{S}_{0}(\tau) \rho=\left(\mathbb{1}+\tau \cdot\left[\mathcal{L}-\left(\mathcal{J}_{1}+\mathcal{J}_{2}\right)\right]\right) \rho+O\left(\tau^{2}\right)
$$

Taking the limit $\tau \rightarrow 0$ we have Eq. (15) as required. 
There are many different definitions of $\mathcal{S}_{0}, \mathcal{J}_{1}$ and $\mathcal{J}_{2}$ that satisfy the above lemma. However, definitions (12) and (14) are somewhat special: the quantities $\rho_{\mathrm{c}}\left(k_{1}, \ldots, k_{m} ; \Delta t\right)$ and $p\left(k_{1}, \ldots, k_{m} ; \Delta t\right)$ which they define have an important physical meaning [12]. Suppose that the continuous measurements were performed over the time interval $\Delta t$ and recorded as a sequence $\left(k_{1}, \ldots, k_{m} ; \Delta t\right)$ of photodetector labels in the order of photodetections. For example, $k_{j}=1$ would mean that the $j$ th photodetection was registered by the first detector. Then the probability of the measurement record $\left(k_{1}, \ldots, k_{m} ; \Delta t\right)$ is given by $p\left(k_{1}, \ldots, k_{m} ; \Delta t\right)$, and the corresponding conditional state is $\rho_{\mathrm{c}}\left(k_{1}, \ldots, k_{m} ; \Delta t\right)$.

We will now prepare to consider the conditional system evolution on time scales large compared to $1 / \mathrm{g}$. First we notice that

$$
H_{\text {int }}=H_{0}+H_{1}
$$

where

$$
\begin{array}{lll}
H_{0} \equiv-g\left(a^{\dagger}+a\right) \sigma_{y} / 2, & & \sigma_{y} \equiv i\left(\sigma^{\dagger}-\sigma\right) \\
H_{1} \equiv i g\left(a^{\dagger}-a\right) \sigma_{x} / 2, & & \sigma_{x} \equiv \sigma^{\dagger}+\sigma .
\end{array}
$$

We define

$$
Q \equiv \exp \left(-i H_{0} t-i H_{1} t+F t\right)
$$

where

$$
F \equiv E\left(a^{\dagger}-a\right)-\gamma a^{\dagger} a / 2
$$

These definitions are connected to the definition (12) of the smooth evolution operator via the relation

$$
N_{0} \equiv e^{-\gamma|\beta|^{2} t / 2} Q
$$

We rewrite $Q$ in the form

$$
Q=e^{-i H_{0} t} R_{0}
$$

so that

$$
\frac{d Q}{d t}=-i H_{0} e^{-i H_{0} t} R_{0}+e^{-i H_{0} t} \frac{d R_{0}}{d t} .
$$

From the definition (22) and Eq. (25) we have:

$$
\frac{d Q}{d t}=\left(F-i H_{0}-i H_{1}\right) e^{-i H_{0} t} R_{0}
$$

Combining the last two equations we obtain that $R_{0}$ obeys the equation

$$
\frac{d R_{0}}{d t}=\left(X(t)+e^{i H_{0} t} F e^{-i H_{0} t}\right) R_{0}
$$

where 


$$
X(t) \equiv-e^{i H_{0} t} i H_{1} e^{-i H_{0} t}
$$

Using the Corollary to Theorem 1 from the Appendix together with the identity $e^{-y a^{\dagger}} a=$ $(a+y) e^{-y a^{\dagger}}$ we obtain

$$
\begin{aligned}
2 X(t) & =g e^{i H_{0} t}\left(a^{\dagger}-a\right) \sigma_{x} e^{-i H_{0} t} \\
& =g\left(a^{\dagger}-a-i g \sigma_{y} t\right) e^{i H_{0} t} \sigma_{x} e^{-i H_{0} t}
\end{aligned}
$$

The identity $e^{i A \sigma_{y}}=\cos A+i \sigma_{y} \sin A$ gives

$$
e^{i H_{0} t} \sigma_{x} e^{-i H_{0} t}=\sigma_{x} \cos \left[g t\left(a^{\dagger}+a\right)\right]-\sigma_{z} \sin \left[g t\left(a^{\dagger}+a\right)\right],
$$

where $\sigma_{z} \equiv i \sigma_{y} \sigma_{x}$. Finally we obtain

$$
2 X(t)=g\left(a^{\dagger}-a-i g \sigma_{y} t\right)\left(\sigma_{x} \cos \left[g t\left(a^{\dagger}+a\right)\right]-\sigma_{z} \sin \left[g t\left(a^{\dagger}+a\right)\right]\right) .
$$

At time scales large compared to $1 / g$ we can neglect terms oscillating at frequency $1 / g$ in Eq. (28). This means we can set $X(t)=0$ in Eq. (28), which becomes

$$
\frac{d R_{0}}{d t} \approx e^{i H_{0} t} F e^{-i H_{0} t} R_{0}
$$

This approximation has some similarity with the standard rotating-wave approximation.

Now consider the operator

$$
M \equiv \exp \left(-i H_{0} t+F t\right)
$$

Using the same technique as in Eqns. (25 - 28) it is easy to show that

$$
M=e^{-i H_{0} t} R_{1}
$$

where $R_{1}$ obeys the equation:

$$
\frac{d R_{1}}{d t}=e^{i H_{0} t} F e^{-i H_{0} t} R_{1}
$$

This equation coincides with (B3), which means that at time scales $\delta t \gg 1 / g$ the operator $Q$ can be replaced with $M$. The smooth evolution $\mathcal{S}_{0}$ can therefore be approximated as

$$
\mathcal{S}_{0} \approx \mathcal{S}
$$

where $\mathcal{S}$ is defined as

$$
\mathcal{S}(t) \rho \equiv N(t) \rho[N(t)]^{\dagger},
$$

and where

$$
N(t) \equiv \exp \left[-i H_{0} t+E\left(a^{\dagger}-a\right) t-\frac{\gamma}{2}\left(a^{\dagger} a+|\beta|^{2}\right) t\right] .
$$




\section{CONDITIONAL EVOLUTION FOR ARBITRARY INITIAL STATES}

In this section we derive a general formula for the state, $\rho_{\mathrm{c}}\left(k_{1}, \ldots, k_{m} ; \Delta t\right)$, conditioned on a discrete photocount record for an arbitrary initial state. The formula is a direct consequence of two technical theorems, whose proofs are given in the Appendix. At time scales $\delta t \gg 1 / g$, the theorems allow us to simplify Eq. (11) by changing the order in which the smooth evolution operators $\mathcal{S}$ and the jump operators $\mathcal{J}_{k}$ appear.

Using these theorems we can proceed with the calculation of the conditional density matrix $\rho_{\mathrm{c}}\left(k_{1}, \ldots, k_{m} ; \Delta t\right)$. We have from Eqns. (11), (14) and Eqns. (37 39) that

$$
\begin{aligned}
& p\left(k_{1}, \ldots, k_{m} ; \Delta t\right) \rho_{\mathrm{c}}\left(k_{1}, \ldots, k_{m} ; \Delta t\right) \\
& \approx \frac{1}{m !} \int_{0}^{\Delta t} d t_{m} \cdots \int_{0}^{\Delta t} d t_{2} \int_{0}^{\Delta t} d t_{1}\left[N\left(\Delta t-t_{m}\right) C_{k_{m}} \cdots N\left(t_{2}-t_{1}\right) C_{k_{1}} N\left(t_{1}\right)\right] \rho_{0}[\cdots]^{\dagger} .
\end{aligned}
$$

We can now use Theorem 2 to compute the operator in the square brackets. We have, for instance,

$$
C_{k_{1}} N\left(t_{1}\right)=N\left(t_{1}\right) f_{k}\left[e^{-\gamma t_{1} / 2} a+\frac{1-e^{-\gamma t_{1} / 2}}{\gamma}\left(2 E+i g \sigma_{y}\right)+(-1)^{k_{1}} \beta\right] .
$$

Then, using the identity $N\left(t_{2}-t_{1}\right) N\left(t_{1}\right)=N\left(t_{2}\right)$, we see that repeating the same type of calculations we have

$$
\begin{aligned}
& N\left(\Delta t-t_{m}\right) C_{k_{m}} \cdots N\left(t_{2}-t_{1}\right) C_{k_{1}} N\left(t_{1}\right)= \\
& N(\Delta t) \prod_{p=1}^{m} f_{k_{p}}\left[e^{-\gamma t_{p} / 2} a+\frac{1-e^{-\gamma t_{p} / 2}}{\gamma}\left(2 E+i g \sigma_{y}\right)+(-1)^{k_{p}} \beta\right] .
\end{aligned}
$$

Using the identity $f_{k} f_{k}^{*}=\gamma / 2$ we therefore have

$$
p\left(k_{1}, \ldots, k_{m} ; \Delta t\right) \rho_{\mathrm{c}}\left(k_{1}, \ldots, k_{m} ; \Delta t\right)=\frac{\gamma^{m}}{2^{m} m !} N(\Delta t) G\left(\rho_{0}, \beta\right) N^{\dagger}(\Delta t),
$$

where

$$
\begin{aligned}
G\left(\rho_{0}, \beta\right)= & \int_{0}^{\Delta t} d t_{m} \int_{0}^{\Delta t} d t_{m-1} \cdots \int_{0}^{\Delta t} d t_{1} \\
& \left(\prod_{p=1}^{m}\left[e^{-\gamma t_{p} / 2} a+\frac{1-e^{-\gamma t_{p} / 2}}{\gamma}\left(2 E+i g \sigma_{y}\right)+(-1)^{k_{p}} \beta\right]\right) \rho_{0}(\cdots)^{\dagger} .
\end{aligned}
$$

For notational convenience, we do not indicate explicitely the dependence of $G\left(\rho_{0}, \beta\right)$ on the measurement record $\left(k_{1}, \ldots, k_{m} ; \Delta t\right)$ which, however, should always be remembered.

Equations (43) and (44) have a relatively simple structure. The terms $N(\Delta t)$, which are given in factored form by Theorem 1 , are the same for all possible measurement records. This means that all the information about the measurement records is contained in the function $G\left(\rho_{0}, \beta\right)$. The integrand in $G\left(\rho_{0}, \beta\right)$ is a polynomial in $a, \sigma_{y}$ and $\rho_{0}$. The scalar coefficients of this polynomial are constants or proportional to either $e^{-\gamma t_{p} / 2}$ or $e^{-\gamma t_{p}}$. Therefore all the integrals in Eq. (44) can be easily evaluated, so that $G\left(\rho_{0}, \beta\right)$ takes the form of a polynomial in $a, \sigma_{y}$ and $\rho_{0}$ with known coefficients. In this way, Eqns. (43) and (44) provide an explicit solution for the conditional evolution on the time scales considered. 


\section{DERIVATION OF THE STEADY STATE}

In this section we show that, at timescales $\delta t \gg 1 / g$, the state $\rho_{\mathrm{ss}}$ defined by (6) is a steady state of the master equation (77). Notice that the only free parameter in our homodyne measurements is the complex parameter $\beta$. If we can find a value of $\beta$ such that for any measurement record $\left(k_{1}, \ldots, k_{m} ; \Delta t\right)$, the conditional density matrix satisfies

$$
\rho_{\mathrm{c}}\left(k_{1}, \ldots, k_{m} ; \Delta t\right)=\rho_{\mathrm{ss}},
$$

then $\rho_{\mathrm{ss}}$ must be a steady state. This is because the solution (10) of the unconditional master equation (7) becomes, in this case,

$$
\rho(\Delta t)=\sum_{m=0}^{\infty} \sum_{k_{1}, \ldots, k_{m}} p\left(k_{1}, \ldots, k_{m} ; \Delta t\right) \rho_{\mathrm{ss}}=\rho_{\mathrm{ss}}
$$

for any $\Delta t$. Intuitively, one would expect that, if subjected to a nontrivial measurement, the system would normally depart from the steady state. In our case, however, we will find that Eq. (45) is satisfied for all real values of $\beta$.

Before we proceed with our rigorous analysis it may be helpful to develop some intuition about the dependence of the conditional evolution on $\beta$. In particular we are interested in the dependence of the conditional evolution on the phase $\phi=\arg \beta$. In our analysis we deal with the conditional evolution conditioned on a discrete photocount record, which is the most general case. However, a lot of insight about the dependence of the conditional evolution on the phase $\phi$ can be gained by taking the limit $|\beta| \rightarrow \infty$. In this limit the detectors are registering continuous photocurrents rather than discrete photocounts. Because the resulting measurement records can be viewed as continuous functions of time it becomes possible to derive a master equation for the conditional density matrix $\rho_{\mathrm{c}}$. According to Ref. [11], this can be done by taking the double limit $|\beta| \propto \epsilon^{-1} \rightarrow \infty$ and $\gamma \Delta t \propto \epsilon^{3 / 2} \rightarrow 0$ in the Dyson expansion (10). If the measurement record consists of the difference photocurrent $I_{-}=I_{2}-I_{1}$, where $I_{1}$ and $I_{2}$ are the photocurrents detected by the first and the second detectors respectively, then the resulting master equation for the conditional density matrix becomes [14, 11

$$
\dot{\rho}_{\mathrm{c}}=\mathcal{L} \rho+\sqrt{\gamma \eta}\left(e^{-i \phi} a \rho_{\mathrm{c}}+e^{i \phi} \rho_{\mathrm{c}} a^{\dagger}-\operatorname{tr}\left[\rho_{\mathrm{c}}\left(e^{-i \phi} a+e^{i \phi} a^{\dagger}\right)\right] \rho_{\mathrm{c}}\right) \xi
$$

where $\eta$ is the efficiency of the photodetection, and $\xi$ is the Gaussian white noise which, in practice, should be taken from experimental observations of the difference photocurrent $I_{-}$ via the relation

$$
I_{-}=|\beta|\left(\gamma \eta \operatorname{tr}\left[\rho_{\mathrm{c}}\left(e^{i \phi} a^{\dagger}+e^{-i \phi} a\right)\right]+\sqrt{\gamma \eta} \xi\right)
$$

Compared to the unconditional master equation (2), Eq. (47) has an additional term

$$
\sqrt{\gamma \eta}\left(e^{-i \phi} a \rho_{\mathrm{c}}+e^{i \phi} \rho_{\mathrm{c}} a^{\dagger}-\operatorname{tr}\left[\rho_{\mathrm{c}}\left(e^{-i \phi} a+e^{i \phi} a^{\dagger}\right)\right] \rho_{\mathrm{c}}\right) \xi
$$

which, for $\phi=0$ and $\rho_{\mathrm{c}}=\rho_{\mathrm{ss}}$, is proportional to $\rho_{\mathrm{ss}}$. This means that, if $\mathcal{L} \rho_{\mathrm{ss}}=0$, i.e., if $\rho_{\mathrm{ss}}$ is a steady state of the unconditional evolution, then conditional and unconditional 
evolution coincide for $\phi=0$. This situation is similar to the one described by Eqns. (45) and (46), which suggests to consider the case of real $\beta$ in the following rigorous derivation.

We now substitute $\rho_{0}=\rho_{\mathrm{ss}}$ from Eq. (6) into Eq. (44), keeping $\beta$ arbitrary for the moment. We obtain

$$
G\left(\rho_{\mathrm{ss}}, \beta\right)=\int_{0}^{\Delta t} d t_{m} \int_{0}^{\Delta t} d t_{m-1} \cdots \int_{0}^{\Delta t} d t_{1}\left(\prod_{p=1}^{m} e^{-\gamma t_{p} / 2}\left[a+f\left(\sigma_{y}, \beta\right)\right]\right) \rho_{\mathrm{ss}}(\cdots)^{\dagger}
$$

where

$$
f\left(\sigma_{y}, \beta\right) \equiv \frac{e^{\gamma t_{p} / 2}-1}{\gamma}\left(2 E+i g \sigma_{y}\right)+(-1)^{k_{p}} \beta e^{\gamma t_{p} / 2}
$$

We note that

$$
\left[\sigma_{y}, \rho_{\mathrm{ss}}\right]=0 \quad \text { and } \quad\left(\sigma_{y}\right)^{2}=\mathbb{1}
$$

Using the first of these properties and the expression for $\rho_{\mathrm{ss}}$ as given by Eq. (6), we have by direct calculation

$$
\begin{aligned}
{\left[a+f\left(\sigma_{y}, \beta\right)\right] \rho_{\mathrm{ss}}\left[a+f\left(\sigma_{y}, \beta\right)\right]^{\dagger}=} & \left(f\left(\sigma_{y}, \beta\right)\left[f\left(\sigma_{y}, \beta\right)\right]^{\dagger}+2 \operatorname{Re}\left[f\left(\sigma_{y}, \beta\right)\right] \operatorname{Re}(\alpha)\right. \\
& \left.+2 \operatorname{Im}\left[f\left(\sigma_{y}, \beta\right)\right] \operatorname{Im}(\alpha) \sigma_{y}+|\alpha|^{2}\right) \rho_{\mathrm{ss}}
\end{aligned}
$$

where $\alpha=(2 E+i g) / \gamma$. We will use this equation for imaginary $\beta$ in the next section.

For the rest of this section, we assume that $\beta$ is real. Using this and the fact that $\left(\sigma_{y}\right)^{2}=\mathbb{1}$, we find that

$$
f\left(\sigma_{y}\right)\left[f\left(\sigma_{y}\right)\right]^{\dagger}=\frac{4 E^{2}+g^{2}}{\gamma^{2}}\left[e^{\gamma t_{p} / 2}-1\right]^{2}+\beta^{2} e^{\gamma t_{p}}+\frac{4 E \beta}{\gamma}(-1)^{k_{p}}\left(e^{\gamma t_{p}}-e^{\gamma t_{p} / 2}\right),
$$

and

$$
\operatorname{Re}\left[f\left(\sigma_{y}\right)\right] \operatorname{Re}(\alpha)+\operatorname{Im}\left[f\left(\sigma_{y}\right)\right] \operatorname{Im}(\alpha) \sigma_{y}=\frac{4 E^{2}+g^{2}}{\gamma^{2}}\left(e^{\gamma t_{p} / 2}-1\right)+\frac{2 E \beta}{\gamma}(-1)^{k_{p}} e^{\gamma t_{p} / 2} .
$$

Because $|\alpha|^{2}=|2 E+i g|^{2} / \gamma^{2}=\left(4 E^{2}+g^{2}\right) / \gamma^{2}$ we therefore have according to Eq. (53):

$$
\left[a+f\left(\sigma_{y}, \beta\right)\right] \rho_{\mathrm{ss}}\left[a+f\left(\sigma_{y}, \beta\right)\right]^{\dagger}=e^{\gamma t_{p}}\left[\frac{4 E^{2}+g^{2}}{\gamma^{2}}+(-1)^{k_{p}} \frac{4 E \beta}{\gamma}+\beta^{2}\right] .
$$

Substituting this into (50) we obtain:

$$
G\left(\rho_{\mathrm{ss}}, \beta\right)=(\Delta t)^{m} \prod_{p=1}^{m}\left(\frac{4 E^{2}+g^{2}}{\gamma^{2}}+(-1)^{k_{p}} \frac{4 E \beta}{\gamma}+\beta^{2}\right) \rho_{\mathrm{ss}} .
$$

Therefore, according to Eq. (43),

$$
\rho_{\mathrm{c}}\left(k_{1}, \ldots, k_{m} ; \Delta t\right) \propto N(\Delta t) \rho_{\mathrm{ss}} N^{\dagger}(\Delta t), \quad \text { for any real } \beta .
$$


As the final step of our argument, we now prove a lemma that, together with Eq. (58) and the normalization of the density matrix, implies Eq. (45).

Lemma 2 Smooth evolution leaves $\rho_{\mathrm{ss}}$ invariant in the following sense:

$$
N(\Delta t) \rho_{\mathrm{ss}} N^{\dagger}(\Delta t) \propto \rho_{\mathrm{ss}} .
$$

\section{Proof}

Because $\sigma_{y}$ and $\rho_{\mathrm{ss}}$ commute, we can see from Eq. (6) that the smooth evolution leaves $\rho_{\mathrm{ss}}$ diagonal:

$$
N(\Delta t) \rho_{\mathrm{ss}}[N(\Delta t)]^{\dagger}=\left(\begin{array}{cc}
\Lambda_{1} & 0 \\
0 & \Lambda_{2}
\end{array}\right)
$$

Using Theorem 1 we have

$$
\begin{aligned}
& 2 e^{-2 Z_{1}} \Lambda_{1}=\left(e^{-\frac{\gamma \Delta t}{2} a^{\dagger} a} e^{Z_{2}^{+} a^{\dagger}} e^{Z_{3}^{-} a}\right)|\alpha\rangle\langle\alpha|(\cdots)^{\dagger}, \\
& 2 e^{-2 Z_{1}} \Lambda_{2}=\left(e^{-\frac{\gamma \Delta t}{2} a^{\dagger} a} e^{Z_{2}^{-} a^{\dagger}} e^{Z_{3}^{+} a}\right)\left|\alpha^{*}\right\rangle\left\langle\alpha^{*}\right|(\cdots)^{\dagger},
\end{aligned}
$$

where

$$
\begin{aligned}
& Z_{2}^{ \pm} \equiv \frac{2 E \pm i g}{\gamma}\left(e^{\gamma \Delta t / 2}-1\right), \\
& Z_{3}^{ \pm} \equiv \frac{2 E \pm i g}{\gamma}\left(e^{-\gamma \Delta t / 2}-1\right) .
\end{aligned}
$$

In order to calculate $\Lambda_{1}$ we use the identity $e^{\lambda a^{\dagger}}|\alpha\rangle\left\langle\alpha\left|e^{\lambda^{*} a}=e^{|\alpha+\lambda|^{2}-|\alpha|^{2}}\right| \alpha+\lambda\right\rangle\langle\alpha+\lambda|$ which gives

$$
2 e^{-2 Z_{1}} \Lambda_{1}=\left|e^{Z_{3}^{-} \alpha}\right| e^{\left|\alpha+Z_{2}^{+}\right|^{2}-|\alpha|^{2}}\left(e^{-\frac{\gamma \Delta t}{2} a^{\dagger} a}\left|\alpha+Z_{2}^{+}\right\rangle\left\langle\alpha+Z_{2}^{+}\right| e^{-\frac{\gamma \Delta t}{2} a^{\dagger} a}\right) .
$$

Now, with the help of the identity $e^{-\lambda a^{\dagger} a}|\alpha\rangle\left\langle\alpha\left|e^{-\lambda a^{\dagger} a}=e^{|\alpha|^{2}\left(e^{-2 \lambda}-1\right)}\right| \alpha e^{-\lambda}\right\rangle\left\langle\alpha e^{-\lambda}\right|$ we have

$$
2 e^{-2 Z_{1}} \Lambda_{1}=\left|e^{Z_{3}^{-} \alpha}\right| e^{\left|\alpha+Z_{2}^{+}\right|^{2} e^{-\gamma \Delta t}-|\alpha|^{2}}\left|\left(\alpha+Z_{2}^{+}\right) e^{-\frac{\gamma \Delta t}{2}}\right\rangle\left\langle\left(\alpha+Z_{2}^{+}\right) e^{-\frac{\gamma \Delta t}{2}}\right| .
$$

Using the definition of $Z_{2}^{+}$and the value of $\alpha=(2 E+i g) / \gamma$ we see that

$$
\left(\alpha+Z_{2}^{+}\right) e^{-\frac{\gamma \Delta t}{2}}=\alpha .
$$

Therefore

$$
2 e^{-2 Z_{1}} \Lambda_{1}=\left|e^{Z_{3}^{-} \alpha}\right||\alpha\rangle\langle\alpha| .
$$

Repeating the same arguments for $\Lambda_{2}$ we have from Eq. (61):

$$
2 e^{-2 Z_{1}} \Lambda_{2}=\left|e^{Z_{3}^{+} \alpha^{*}}\right|\left|\alpha^{*}\right\rangle\left\langle\alpha^{*}\right|
$$

Because $\left|e^{Z_{3}^{-} \alpha}\right|=\left|e^{Z_{3}^{+} \alpha^{*}}\right|$ we can now see that

$$
\left(\begin{array}{cc}
\Lambda_{1} & 0 \\
0 & \Lambda_{2}
\end{array}\right) \propto\left(\begin{array}{cc}
|\alpha\rangle\langle\alpha| & 0 \\
0 & \left|\alpha^{*}\right\rangle\left\langle\alpha^{*}\right|
\end{array}\right)=2 \rho_{\mathrm{ss}} .
$$

Together with Eq. (60) this completes the proof. 


\section{CONDITIONAL EVOLUTION STARTING FROM THE STEADY STATE}

In the previous section we have shown that, for a real value of $\beta$, a homodyne measurement does not give any information about the system once it has reached the steady state $\rho_{\mathrm{ss}}$. Although this fact was useful in confirming that $\rho_{\mathrm{ss}}$ is indeed a steady state of the system, such a measurement would be pointless in practice.

We therefore consider the case of purely imaginary $\beta$, for which the homodyne measurement does provide information about the system. We write $\beta$ in the form $\beta=i \beta_{0}$, where $\beta_{0}$ is real. To find the conditional density matrix in this case, we go back to Eqns. (50 53) and obtain by direct calculation:

$$
f\left(\sigma_{y}\right)\left[f\left(\sigma_{y}\right)\right]^{\dagger}=\frac{4 E^{2}+g^{2}}{\gamma^{2}}\left(e^{\gamma t_{p} / 2}-1\right)^{2}+\beta_{0}^{2} e^{\gamma t_{p}}+(-1)^{k_{p}} \frac{2 g \beta_{0}}{\gamma}\left(e^{\gamma t_{p}}-e^{\gamma t_{p} / 2}\right),
$$

and

$$
\operatorname{Re}\left[f\left(\sigma_{y}\right)\right] \operatorname{Re}(\alpha)+\operatorname{Im}\left[f\left(\sigma_{y}\right)\right] \operatorname{Im}(\alpha) \sigma_{y}=\frac{4 E^{2}+g^{2}}{\gamma^{2}}\left(e^{\gamma t_{p} / 2}-1\right)+(-1)^{k_{p}} \frac{g \beta_{0}}{\gamma} e^{\gamma t_{p} / 2} \sigma_{y} .
$$

Therefore

$$
\begin{array}{rl}
G\left(\rho_{\mathrm{ss}}, i \beta_{0}\right)=\int_{0}^{\Delta t} & d t_{m} \int_{0}^{\Delta t} d t_{m-1} \cdots \int_{0}^{\Delta t} d t_{1} \\
& \prod_{p=1}^{m}\left(\frac{4 E^{2}+g^{2}}{\gamma^{2}}+\beta_{0}^{2}+(-1)^{k_{p}} \frac{2 g \beta_{0}}{\gamma}\left[\mathbb{1}+\left(\sigma_{y}-\mathbb{1}\right) e^{-\gamma t_{p} / 2}\right]\right) \rho_{\mathrm{ss}} .
\end{array}
$$

Performing the integration we obtain

$$
\begin{aligned}
G\left(\rho_{\mathrm{ss}}, i \beta_{0}\right)=\prod_{p=1}^{m}\left[\Delta t \cdot \left(\frac{4 E^{2}+g^{2}}{\gamma^{2}}\right.\right. & \left.+\beta_{0}^{2}+(-1)^{k_{p}} \frac{2 g \beta_{0}}{\gamma}\right) \\
& \left.+(-1)^{k_{p}} 4 g \beta_{0}\left(\sigma_{y}-\mathbb{1}\right) \frac{1-e^{-\gamma \Delta t / 2}}{\gamma^{2}}\right] \rho_{\mathrm{ss}} .
\end{aligned}
$$

Because $\rho_{\mathrm{ss}}$ and $\sigma_{y}$ are both diagonal in the basis $\{| \pm\rangle\}$ defined in Eq. (舟), the conditional density matrix can be written in the form

$$
\rho_{\mathrm{c}}\left(k_{1}, \ldots, k_{m} ; \Delta t\right)=\left(\begin{array}{cc}
\lambda_{1}|\alpha\rangle\langle\alpha| & 0 \\
0 & \lambda_{2}\left|\alpha^{*}\right\rangle\left\langle\alpha^{*}\right|
\end{array}\right)
$$

where $\alpha=(2 E+i g) / \gamma$. For the eigenvalues $\lambda_{1}$ and $\lambda_{2}=1-\lambda_{1}$ we have the following simple formula:

$$
\frac{\lambda_{1}}{\lambda_{2}}=\prod_{p=1}^{m} \frac{b+(-1)^{k_{p}}}{b+(-1)^{k_{p}}\left(1-\frac{4}{\gamma \Delta t}\left(1-e^{-\gamma \Delta t / 2}\right)\right)}
$$

where $b \equiv\left(4 E^{2}+g^{2}+\gamma^{2} \beta_{0}^{2}\right) /\left(2 g \gamma \beta_{0}\right)$. Similarly simple expressions can be obtained for any complex reference field $\beta$. 


\section{CONCLUSION}

In this paper, we have given explicit formulas for the quantum state evolution conditioned on a discrete homodyne photocount record for a typical experimental setup in single-atom cavity QED. These formulas have potential applications for the real-time processing of experimental data. The general methods developed here can be applied to a wide class of similar systems. For example, it should be straightforward to generalize our results to the case of heterodyne measurements.

\section{ACKNOWLEDGMENTS}

We would like to thank Howard Wiseman for helpful comments on a previous version of this manuscript. This work was supported by the EU IST programme.

\section{APPENDIX}

Theorem 1 The operator

$$
M(t)=\exp \left[i g \frac{\sigma_{y}}{2}\left(a^{\dagger}+a\right) t+E\left(a^{\dagger}-a\right) t-\frac{\gamma t}{2} a^{\dagger} a\right]
$$

can be factorized as

$$
M(t)=e^{Z_{1}} e^{-\frac{\gamma t}{2} a^{\dagger} a} e^{Z_{2} a^{\dagger}} e^{Z_{3} a}
$$

where

$$
\begin{aligned}
& Z_{1}=\frac{4 E^{2}+g^{2}}{\gamma^{2}}\left(1-e^{-\gamma t / 2}-\gamma t / 2\right) \\
& Z_{2}=\frac{2 E+i g \sigma_{y}}{\gamma}\left(e^{\gamma t / 2}-1\right) \\
& Z_{3}=\frac{2 E-i g \sigma_{y}}{\gamma}\left(e^{-\gamma t / 2}-1\right)
\end{aligned}
$$

\section{Proof}

Because $a, a^{\dagger}, a^{\dagger} a$ and $\mathbb{1}$ span a Lie algebra, $M(t)$ can be factorized in a systematic way as follows. First we find a function $x(t)$ such that

$$
M(t)=e^{x(t) a^{\dagger} a} \tilde{M}(t)
$$

where $\tilde{M}(t)$ is an exponential of a linear combination of $a$ and $a^{\dagger}$. We will then repeat the same procedure factorizing $\tilde{M}$ which will conclude the prove of the theorem.

Equation (78) gives

$$
\frac{d M}{d t}=\dot{x} a^{\dagger} a e^{x a^{\dagger} a} \tilde{M}+e^{x a^{\dagger} a} \frac{d \tilde{M}}{d t}
$$


On the other hand, Eq. (75) gives

$$
\frac{d M}{d t}=\left[i g \frac{\sigma_{y}}{2}\left(a^{\dagger}+a\right)+E\left(a^{\dagger}-a\right)-\frac{\gamma}{2} a^{\dagger} a\right] e^{x a^{\dagger} a} \tilde{M}
$$

Comparing this expression with the previous one we have

$$
\frac{d \tilde{M}}{d t}=\left[\chi(x)-(\dot{x}+\gamma / 2) a^{\dagger} a\right] \tilde{M}
$$

where

$$
\chi(x)=e^{-x a^{\dagger} a}\left[i g \frac{\sigma_{y}}{2}\left(a^{\dagger}+a\right)+E\left(a^{\dagger}-a\right)\right] e^{x a^{\dagger} a} .
$$

Using the identity $e^{-x a^{\dagger} a} a e^{x a^{\dagger} a}=a e^{x}$, the above equation can be rewritten as

$$
\chi(x)=\left(E+i g \frac{\sigma_{y}}{2}\right) e^{-x} a^{\dagger}-\left(E-i g \frac{\sigma_{y}}{2}\right) e^{x} a .
$$

Looking at Eq. (81) we demand that

$$
\dot{x}+\gamma / 2=0
$$

thereby making $d \tilde{M} / d t$ independent of $a^{\dagger} a$. From Eq. (75) we see that $M(0)=\mathbb{1}$ and therefore, we choose, in accordance with Eq. (78), that

$$
x(0)=0 \text { and } \tilde{M}(0)=\mathbb{1} .
$$

With these conditions equation (84) can be integrated to give, according to Eqns. (78) and (81),

$$
M(t)=e^{-\frac{\gamma t}{2} a^{\dagger} a} \tilde{M}(t)
$$

where

$$
\frac{d \tilde{M}}{d t}=\left[\left(E+i g \frac{\sigma_{y}}{2}\right) e^{\gamma t / 2} a^{\dagger}-\left(E-i g \frac{\sigma_{y}}{2}\right) e^{-\gamma t / 2} a\right] e^{y a^{\dagger}} \tilde{M}^{\prime}
$$

The proof of the theorem will be completed if we repeat the same procedure for factorizing $\tilde{M}$. As before we introduce a function $y(t)$ such that

$$
\tilde{M}(t)=e^{y(t) a^{\dagger}} \tilde{M}^{\prime}(t)
$$

We therefore have

$$
\frac{d \tilde{M}}{d t}=\dot{y} a^{\dagger} e^{y a^{\dagger}} \tilde{M}^{\prime}+e^{y a^{\dagger}} \frac{d \tilde{M}^{\prime}}{d t}
$$

Combined with Eq. (87) this gives

$$
\frac{d \tilde{M}^{\prime}}{d t}=\left[\left(E+i g \frac{\sigma_{y}}{2}\right) e^{\gamma t / 2} a^{\dagger}-\left(E-i g \frac{\sigma_{y}}{2}\right) e^{-\gamma t / 2} e^{-y a^{\dagger}} a e^{y a^{\dagger}}-\dot{y} a^{\dagger}\right] \tilde{M}^{\prime} .
$$


Using the identity $e^{-y a^{\dagger}} a e^{y a^{\dagger}}=a+y$ we rewrite the above expression as

$$
\frac{d \tilde{M}^{\prime}}{d t}=\left(\left[\left(E+i g \frac{\sigma_{y}}{2}\right) e^{\gamma t / 2}-\dot{y}\right] a^{\dagger}-\left(E-i g \frac{\sigma_{y}}{2}\right) e^{-\gamma t / 2}(a+y)\right) \tilde{M}^{\prime} .
$$

We eliminate $a^{\dagger}$ from this expression by setting

$$
\dot{y}=\left(E+i g \frac{\sigma_{y}}{2}\right) e^{\gamma t / 2} .
$$

Equation (88) suggests the boundary conditions

$$
y(0)=0 \text { and } \tilde{M}^{\prime}(0)=\mathbb{1} .
$$

Performing integration in (91) and in (92) using these boundary conditions and the fact that $\sigma_{y}^{2}=\mathbb{1}$ we have according to 88 )

$$
\tilde{M}(t)=\exp \left[\frac{2 E+i g \sigma_{y}}{\gamma}\left(e^{\gamma t / 2}-1\right) a^{\dagger}\right] \tilde{M}^{\prime}(t)
$$

where

$$
\tilde{M}^{\prime}(t)=\exp \left[\frac{4 E^{2}+g^{2}}{\gamma^{2}}\left(1-e^{-\gamma t / 2}-\gamma t / 2\right)\right] \exp \left[-\frac{2 E-i g \sigma_{y}}{\gamma}\left(1-e^{-\gamma t / 2}\right) a\right] .
$$

This completes the proof of the theorem.

\section{Corollary}

$$
e^{-i H_{0} t}=e^{-g^{2} t^{2} / 8} e^{i g t \sigma_{y} a^{\dagger} / 2} e^{i g t \sigma_{y} a / 2}
$$

\section{Proof}

This can be established easily by repeating the arguments of Theorem 1 for $E=0$ and $\gamma=0$.

Theorem 2 Using the definition

$$
f_{k} \equiv \sqrt{\gamma / 2} e^{i \pi(k-1) / 2}
$$

and the notation of Theorem 1, we have

$$
C_{k} M(t)=M(t) f_{k}\left[e^{-\gamma t / 2} a+\frac{1-e^{-\gamma t / 2}}{\gamma}\left(2 E+i g \sigma_{y}\right)+(-1)^{k} \beta\right] .
$$

\section{Proof}

By definition [Eqns. (14) and (97)] and using Theorem 1 we have

$$
C_{k} M(t)=f_{k} e^{Z_{1}}\left[a+(-1)^{k} \beta\right] e^{-\frac{\gamma t}{2} a^{\dagger} a} e^{Z_{2} a^{\dagger}} e^{Z_{3} a},
$$

where $Z_{1}, Z_{2}$ and $Z_{3}$ are specified in the statement of Theorem 1 . Using subsequently the identities $e^{-x a^{\dagger} a} a e^{x a^{\dagger} a}=a e^{x}$ and then $e^{-y a^{\dagger}} a e^{y a^{\dagger}}=a+y$ we have 


$$
\begin{aligned}
C_{k} M(t) & =f_{k} e^{Z_{1}} e^{-\frac{\gamma t}{2} a^{\dagger} a}\left[e^{-\gamma t / 2} a+(-1)^{k} \beta\right] e^{Z_{2} a^{\dagger}} e^{Z_{3} a} \\
& =f_{k} e^{Z_{1}} e^{-\frac{\gamma t}{2} a^{\dagger} a} e^{Z_{2} a^{\dagger}}\left[e^{-\gamma t / 2}\left(a+Z_{2}\right)+(-1)^{k} \beta\right] e^{Z_{3} a} \\
& =f_{k} M(t)\left[e^{-\gamma t / 2}\left(a+Z_{2}\right)+(-1)^{k} \beta\right]
\end{aligned}
$$

Putting the value of $Z_{2}$ from Theorem 1 we have Eq. (98) as required.

[1] C. J. Hood et al., Science 287, 1447 (2000).

[2] P. W. H. Pinkse, T. Fischer, P. Maunz, and G. Rempe, Nature 404, 365 (2000).

[3] X. M. Liu, M. Hug, and G. J. Milburn, Phys. Rev. A 62, 043801 (2000).

[4] A. C. Doherty et al., Phys. Rev. A 62, 012105 (2000).

[5] A. Steane, Rep. Prog. Phys. 61, 117 (1998).

[6] C. J. Hood, M. S. Chapman, T. W. Lynn, and H. J. Kimble, Phys. Rev. Lett. 80, 4157 (1998).

[7] G. Rempe, Phys. World 13, 37 (2000).

[8] M. B. Plenio and P. L. Knight, Rev. Mod. Phys. 70, 101 (1998).

[9] P. Alsing and H. J. Carmichael, Quantum Opt. 3, 13 (1991).

[10] H. Mabuchi and H. M. Wiseman, Phys. Rev. Lett. 81, 4620 (1998).

[11] H. M. Wiseman and G. J. Milburn, Phys. Rev. A 47, 642 (1993).

[12] H. J. Carmichael, An Open Systems Approach to Quantum Optics (Springer, Berlin, 1993).

[13] W. H. Louisell, Quantum Statistical Properties of Radiation (Wiley, New York, 1990).

[14] H. M. Wiseman and G. J. Milburn, Phys. Rev. A 47, 1652 (1993). 